\title{
Novel methods to prevent HCV transmission
}

\author{
Ana Maria Ortega-Prieto ${ }^{1} \&$ Marcus Dorner ${ }^{*}, 1$ \\ ${ }^{1}$ Section of Virology, Department of Medicine, Imperial College London, London, UK \\ * Author for correspondence: Tel.: +44 207594 5037; m.dorner@imperial.ac.uk
}

First draft submitted: 9 June 2017; Accepted for publication: 14 June 2017; Published online: 29 September 2017

Keywords: hepatitis $C$ virus $\bullet$ prevention $\bullet$ treatment $\bullet$ vaccine

Hepatitis $\mathrm{C}$ virus (HCV) is a global health concern with more than 70 million people chronically infected [1]. Persistent immune activation during infection results in progressive liver injury, which, if left untreated may result in liver fibrosis, cirrhosis and hepatocellular carcinoma [2]. Historically, the treatment options for HCV have been plagued by low efficiency and high side-effect profiles, resulting in large numbers of patients living with chronic HCV infection [3]. However, the recent introduction of potent direct-acting antivirals (DAA) has shifted HCV infection to an easily manageable disease, for which a plethora of treatment options is available [4]. However, due to its high cost, availability of treatment is presently limited throughout the world, which is unlikely to change in the coming years. Moreover, HCV infection usually does not result in protective immunity, making re-infection a uniform problem for the control of the HCV epidemic [5]. Especially in light of pre-existing as well as treatment-induced resistance to direct-acting antiviral drugs indicated by resistance-associated substitutions (RAS), it remains unclear as to what contribution these drug-resistant HCV strains are going to have on the landscape to HCV strain transmission. Additionally, most data on the impact of RAS on sustained virological responses arise from highly selected clinical trials. To date, real life data on treatment efficiency and the role of drug resistance in difficult-to-treat populations remain elusive. This, combined with the current lack of a protective vaccine is likely going to impact the ability to eradicate HCV infection, especially in resource-limited settings, demanding an ongoing commitment to re-treat patients. In order to ultimately achieve the goal to sustainably prevent HCV transmission and ultimately eradicate HCV, the development of a vaccine, which offers cross-genotype protection or the introduction of pre-exposure prophylaxis (PrEP) will have to be prioritized. Due to the current high costs of antiviral therapy, the role of PrEP in HCV will likely be very limited, unless generic direct-acting antiviral drugs make PrEP a viable strategy in preventing HCV transmission. Thus, the development and evaluation of HCV vaccine candidates will have to be expedited to ensure efficient prevention of future HCV transmissions.

As we have learned from experiments conducted on chimpanzees as well as observational studies in humans, a broad and sustained T-cell response is a key to immune-mediated clearance of HCV. In chimpanzee models, this resulted in the induction of protective immunity against homologous as well as heterologous HCV challenge [6]. However, human studies show that re-infection with both, homologous as well as heterologous HCV genotypes is likely and strongly associated with the host genetic background [7]. Thus, a viable $\mathrm{HCV}$ vaccine candidate would require the induction of neutralizing antibodies in addition to eliciting broad T-cell responses to ensure universal protection.

Over the past years, several HCV vaccine candidates have been described [8,9]. However, many of the early vaccine candidates failed to confer cross-genotype vaccine protection and the recent ban on experimental infections of chimpanzees with HCV has complicated the evaluation of vaccine candidates in in vivo model systems. Human clinical vaccine trials with several HCV vaccine candidates have been performed in the past and have described the sustained induction of immune responses [10-13]. However, these vaccine trials are usually conducted in healthy volunteers and due to the low probability of HCV transmission in these cohorts, evaluation of vaccine efficacy remains a major challenge for vaccine licensing. Several other infectious diseases, including cholera, typhoid, malaria, influenza, norovirus and dengue virus, have recently attracted attention for conducting human challenge vaccine studies. Since HCV infection is restricted to humans, the introduction of controlled human challenges to test vaccine efficacy would certainly offer an attractive model. However, the development of drug resistance and 
the uncertainty of optimal drug combinations for patients with RAS against multiple DAA mandates caution with such trials due to the chronic nature of HCV infection.

Despite recent advances in the model systems for studying HCV infection [14], most notably the ability to study patient-derived viral isolates [15] and a small animal model for HCV entry in genetically humanized mice [16], the field is still restricted in evaluating vaccine candidates in terms of inducing sterilizing immunity or the impact of resistance-associated substitutions on immune responses. Even though antibody-mediated protection can still be evaluated [16], T-cell-mediated protection and efficacy of vaccine candidates in a full infection model is now restricted to human clinical trials, in which challenges are not feasible. Thus, more focus has to be given to rational vaccine design in order to evaluate only the most promising vaccine candidates as well as the improvement of model systems to study these.

Many of these advanced vaccine design approaches have resulted in promising novel ideas in vaccinology, including the design of vaccine candidates based on the analysis of broadly neutralizing antibodies, which are capable of neutralizing all known HCV genotypes at very low concentrations [17-20]. Combining these approaches based on the induction of humoral immune responses with potent vaccine candidates targeting dendritic cells and T-cell immunity may hold the best chance for producing a broadly cross-genotype protective HCV vaccine.

Irrespective of these developments, access to better model systems allowing the study of immune protection against clinical isolates will be paramount in moving any vaccine candidate to licensing. Current in vivo models still do not enable the challenge with pan-genotypic HCV isolates in the presence of a completely functional immune system. Even though several approaches have resulted in small animal models recapitulating the HCV life cycle using human xenograft models or genetically humanized mice, immune responses in these models still differ significantly from those observed in humans. Optimizing these models to establish challenge models mimicking human immune responses will require further research, independent of whether the final model will constitute a murine or xenograft model. Unfortunately, the introduction of DAA with high cure rates has impacted HCV research in the past years, since the general perception shifted toward a view of having solved the problem of HCV infection. However, it is increasingly evident that re-infection might pose a significant risk, not only in regards to spreading a chronic viral infection, which often goes unnoticed for years but especially for healthcare systems, for which re-treatment of HCV infection poses a significant strain of often already stretched resources. Thus, it is worthwhile revisiting whether further HCV research and funding thereof should be allocated to further benefit patient welfare and develop potent means of preventing HCV infection.

\section{Financial \& competing interests disclosure}

The authors have no relevant affiliations or financial involvement with any organization or entity with a financial interest in or financial conflict with the subject matter or materials discussed in the manuscript. This includes employment, consultancies, honoraria, stock ownership or options, expert testimony, grants or patents received or pending, or royalties.

No writing assistance was utilized in the production of this manuscript.

\section{References}

1. Manns MP, Buti M, Gane E et al. Hepatitis C virus infection. Nat. Rev. Dis. Primers 3, 17006 (2017).

2. Bandiera S, Billie Bian C, Hoshida Y, Baumert TF, Zeisel MB. Chronic hepatitis C virus infection and pathogenesis of hepatocellular carcinoma. Curr. Opin. Virol. 20, 99-105 (2016).

3. Zeuzem S. Interferon-based therapy for chronic hepatitis C: current and future perspectives. Nat. Clin. Pract. Gastroenterol. Hepatol. 5 , 610-622 (2008).

4. Hezode C. Pan-genotypic treatment regimens for hepatitis C virus: advantages and disadvantages in high- and low-income regions. J. Viral Hepat. 24, 92-101 (2017).

5. Scott N, Hellard M, McBryde ES. Modeling hepatitis $C$ virus transmission among people who inject drugs: assumptions, limitations and future challenges. Virulence 7, 201-208 (2016).

6. Major ME et al. Previously infected and recovered chimpanzees exhibit rapid responses that control hepatitis $\mathrm{C}$ virus replication upon rechallenge. J. Virol. 76, 6586-6595 (2002).

7. Sacks-Davis R et al. Hepatitis C virus reinfection and spontaneous clearance of reinfection - the InC3 study. J. Infect. Dis. 212, 1407-1419 (2015).

8. Freeman ZT, Cox AL. Lessons from nature: understanding imunity to HCV to guide vaccine design. PLoS Pathog. 12, e1005632 (2016).

9. Fauvelle C, Colpitts CC, Keck ZY, Pierce BG, Foung SK, Baumert TF. Hepatitis C virus vaccine candidates inducing protective neutralizing antibodies. Exp. Rev. Vacc. 15, 1535-1544 (2016). 
10. Young AM, Stephens DB, Khaleel HA, Havens JR. Hepatitis C vaccine clinical trials among people who use drugs: potential for participation and involvement in recruitment. Contemp. Clin. Trials 41, 9-16 (2015).

11. Swadling $L$ et al. A human vaccine strategy based on chimpanzee adenoviral and MVA vectors that primes, boosts, and sustains functional HCV-specific T-cell memory. Sci. Transl. Med. 6, 261 ra153 (2014).

12. Drane $\mathrm{D}$ et al. Priming of $\mathrm{CD}^{+}$and $\mathrm{CD}^{+}$T-cell responses using a HCV core ISCOMATRIX vaccine: a Phase I study in healthy volunteers. Hum. Vacc. 5, 151-157 (2009).

13. Frey SE et al. Safety and immunogenicity of HCV E1E2 vaccine adjuvanted with MF59 administered to healthy adults. Vaccine 28 , 6367-6373 (2010).

14. Ortega-Prieto AM, Dorner M. The expanding toolbox for hepatitis C virus research. J. Viral Hepat. 23, 320-329 (2016).

15. Saeed M et al. SEC14L2 enables pan-genotype HCV replication in cell culture. Nature 524, 471-475 (2015).

16. Dorner $\mathrm{M}$ et al. A genetically humanized mouse model for hepatitis $\mathrm{C}$ virus infection. Nature 474, 208-211 (2011).

17. de Jong YP et al. Broadly neutralizing antibodies abrogate established hepatitis C virus infection. Sci. Transl. Med. 6, 254 ra129 (2014).

18. Giang E et al. Human broadly neutralizing antibodies to the envelope glycoprotein complex of hepatitis C virus. Proc. Natl Acad. Sci. USA 109, 6205-6210 (2012).

19. Naik AS et al. Reverse epitope mapping of the E2 glycoprotein in antibody associated hepatitis C virus. PLoS ONE 12, e0175349 (2017).

20. Desombere I et al. Monoclonal anti-envelope antibody AP33 protects humanized mice against a patient-derived hepatitis $\mathrm{C}$ virus challenge. Hepatology 63, 1120-1134 (2016). 
